\title{
Pain and mobility improvement and MDA plasma levels in degenerative osteoarthritis, low back pain, and rheumatoid arthritis after infrared A-irradiation ${ }^{\star}$
}

\author{
Werner Siems ${ }^{1 凶}$, Nikolaus Bresgen², Rainer Brenke3, Renate Siems', Manfred Kitzing1, \\ Heike Harting ${ }^{1}$ and Peter M. Eckl ${ }^{2}$
}

1KortexMed Bad Harzburg, Research Institute of Physiotherapy and Gerontology, Bad Harzburg, Germany; ${ }^{2}$ University of Salzburg, Department of Cell Biology, Salzburg, Austria; ${ }^{3}$ Hufeland-Hospital, Center for Pneumology, Allergology, Natural Medicine and Orthopaedics, Bad Ems, Germany

Infrared (IR)-A irradiation can be useful in back and musculoskeletal pain therapy. In this study joint and vertebral column pain and mobility were measured during two weeks of IR-A irradiation treatment of patients suffering from degenerative osteoarthritis of hip and knee, low back pain, or rheumatoid arthritis. Additionally, before and after IR-A treatment MDA serum levels were measured to check if MDA variations accompany changes in pain intensity and mobility. Two-hundred and seven patients were divided into verum groups getting IR-irradiation, placebo groups getting visible, but not IR irradiation, and groups getting no irradiation. In osteoarthritis significant pain reduction according to Visual Analogue Scale and mobility improvements occurred in the verum group. Even though beneficial mean value changes occurred in the placebo group, the improvements in the placebo and No Irradiation groups were without statistical significance. In low back pain, pain and mobility improvements (by 35-40\%) in the verum group were found, too. A delayed (2nd week) mobility improvement in rheumatoid arthritis was seen. However, pain relief was seen immediately. In patients suffering from low back pain or rheumatoid arthritis, the pain and mobility improvements were accompanied by significant changes of MDA serum levels. However, MDA appears not a sensitive biofactor for changes of the pain intensity in degenerative osteoarthritis. Nevertheless, unaffected or lowered MDA levels during intensive IR-A therapy argue against previous reports on free radical formation upon infrared. In conclusion, rapid beneficial effects of IR-A towards musculoskeletal pain and joint mobility loss were demonstrated.

Keywords: infrared, osteoarthritis, rheumatoid arthritis, pain, malondialdehyde (MDA), lipid peroxidation

Received: 11 December, 2009; revised: 26 June, 2010; accepted: 16 August, 2010; available on-line: 08 September, 2010

\section{INTRODUCTION}

Pain is an unpleasant sensory and emotional experience associated with actual or potential tissue damage, or described in terms of such damage (Merskey \& Watson, 1979; Merskey, 1989). The most frequent pain types we suffer from are muscleache, jointpain, backache, neck and shoulder ache, and headache, whereas stomach pain, menstrual pain and toothache occur more seldom. With- out any doubt the occurrence and therapy of pain play an increasing role in modern clinical medicine, both in prevention, therapy, and rehabilitation.

According to statistical data (Health Statistics for U.S. Adults: National Health Interview Survey 2002), in the year $200232.2 \%$ of US citizens aged from 45 to 65 years suffer from painful chronic joint diseases. In $28.8 \%$ degenerative osteoarthrosis was diagnosed. The trend has been increasing in the last years. Already in $200334.9 \%$ US citizens aged from 45 to 65 years suffered from painful chronic joint diseases and 30.0\% from degenerative osteoarthosis (Sondik et al., 2005). Additionally, 13\% of working time was lost due to pain in back or joints (Stewart et al., 2003). Thirty-one million US citizens suffer from low back pain. The yearly costs in the United States directly or indirectly due to back pain were estimated at about 50 billion US\$. Only the costs for analgetic drugs were 25 billion US\$. The European statistics are similar to the data from the United States. The costs of therapy of acute and chronic pain contribute to the budget explosion in the health systems not only of Western countries, but also of the Asian, South and Middle American ones in parallel with the accelerating industrialization. The causal factors for this development are misloading and unbalanced loading in working process, physical and mental stress, but also overweight due to faulty nutrition and progressive lack of movement and exercise.

What alternatives are available? In many cases they include prevention, therapy, and rehabilitation by means of physical medicine, ergonomic optimization, stress handling and stress reduction, improved nutrition and exercise. For pain prevention and therapy warmth applications are widely accepted (Masuda et al., 2005). IR-irradiation is one of the warmth application regimes which can be useful for pain relief, together with hot packs, paraffin, fluid therapy, sauna, diathermy, warmth plasters, gels, and massage crèmes. Warmth applications including IR irradiation have been used for pain therapy for many years. Only in acute inflammation one should avoid warmth.

\footnotetext{
e-mail: siems@kortexmed.de

* The study was carried out within the European project COST-B35 Lipid peroxidation-associated diseases.

Abbreviations: HNE, 4-hydroxynonenal, 4-hydroxy-2,3-trans-nonenal; HPLC, high performance liquid chromatography; IR-A, infrared A; LPO, lipid peroxidation; MDA, malondialdehyde; RA, rheumatoid arthritis; TBA, thiobarbituric acid; VAS, visual analogue pain scale
} 
Various IR wavelengths have different skin penetration characteristics. Only IR-A can reach the circulated layers of subcutis and, therefore, increase the local circulation of muscle tissue. IR-B and IR-C are absorbed in the very superficial layers of epidermis and dermis with their high content of heat nociceptors. The resulting heat pain will limit the irradiation with high $\mathrm{B}$ or $\mathrm{C}$ content. Only at high IR-A content one can use high total intensity of IR irradiation, which leads to a significant pain reduction in parallel to the local increase of circulation. The IR devices of the series InfraCare (Philips, Netherlands) produce IR with $45-50 \%$ of IR-A. These devices are useful for home applications, too. Therefore they offer new possibilities for broad medical self-application if recommended by physicians and physiotherapists.

In this randomised controlled study of patients suffering from degenerative osteoarthritis of hip and knee, low back pain, or rheumatoid arthritis joint or vertebral column pain and mobility were measured during two weeks of IR-A irradiation treatment using InfraCare equipment. Additionally to the clinical data of pain and mobility MDA serum levels were measured before and after IR-A treatment to see if there are parallel changes of pain intensity and MDA. It is well known that nociception and pain stimulation are closely connected with lipid peroxidation (Rokyta et al., 2003; Smith, 2006). In musculoskeletal disorders increased lipid peroxidation has been demonstrated due to inflammation, but also to non-inflammatory factors such as pain (Bagis et al., 2005; Ozgocmen et al., 2006).

\section{MATERIAL AND METHODS}

Groups of patients. Two-hundred and seven patients were involved in the study. Patients suffering from degenerative osteoarthritis (coxarthrosis or gonarthrosis), low back pain, and rheumatoid arthritis were divided in three groups: a) Verum (infrared radiation), b) Placebo (visible radiation only, no infrared), and c) No Irradiation. The study was carried out between April and October 2006. The patients who were irradiated were randomly assigned to the Verum or Placebo group. The Infrared and Placebo emitters were double-blinded (for patient and physiotherapist). In Table 1 numbers of patients in the various groups are given, as well as the age and gender distribution, number of treatments, total time of radiation within the two weeks of treatment, and the mean time of radiation. In general, the recommended mean time of daily irradiation was $15 \mathrm{~min}$ to $30 \mathrm{~min}$.

Equipment for irradiation, recommendations for application, and exclusion of patients. Patients were treated for two weeks with InfraCare HP 3631 and HP 3641 appliances (Philips, Netherlands). We accepted within the pilot study the following recommendations of producing company: The treatment duration approx. $15 \mathrm{~min}$ per session at indicated distance $(30-50 \mathrm{~cm})$; the equipment in general can be used safely several times a day; it can be used every day; IR is not recommended if the patient has a swelling or an inflammation, as heat could aggravate the complaints; painkillers in high dosages can reduce the sensitivity to heat, therefore patients taking painkillers should especially pay attention to keeping the recommended distance between skin and lamp; prolonged IR exposure may damage the eyes, so one is advised not to look directly into the lamp.

We excluded, in accordance with the above recommendations, patients with the following diseases: heart insufficiency (NYHA III and IV), severe disturbances of heart rhythm, myocardial infarction in acute phase, severe respiratory insufficiency, tuberculosis, acute phase of rheumatoid arthritis, acute phase of multiple sclerosis, patients treated with coumarines, deep venous thrombosis, thrombophlebitis, and patients suffering from severe diabetes, thyreotoxic crisis, uraemia, haemolytic anaemias, and porphyry.

Table 1. Characterization of Verum, Placebo, and No Irradiation groups

\begin{tabular}{|c|c|c|c|c|c|c|c|c|}
\hline Diagnosis group & Subgroup & $\begin{array}{l}\text { No. of } \\
\text { patients }\end{array}$ & $\begin{array}{l}\text { Age of } \\
\text { patients }\end{array}$ & Male & Female & $\begin{array}{l}\text { No. of } \\
\text { radiations }\end{array}$ & $\begin{array}{l}\text { Total time of } \\
\text { IR radiation }\end{array}$ & $\begin{array}{l}\text { Mean time of } \\
\text { single } \\
\text { IR radiation }\end{array}$ \\
\hline Dimension & & & years & & & & minutes & minutes \\
\hline \multirow[t]{4}{*}{ Coxarthrosis } & Total group & 50 & 61.8 & 19 & 31 & & & \\
\hline & Verum (IR-A) & 19 & 62.6 & 7 & 12 & 9.0 & 155 & 17.2 \\
\hline & Placebo & 14 & 57.2 & 5 & 9 & 10.5 & 175 & 16.6 \\
\hline & No Irradiation & 17 & 64.6 & 7 & 10 & no & no & no \\
\hline \multirow[t]{4}{*}{ Gonarthrosis } & Total group & 56 & 66.7 & 30 & 26 & & & \\
\hline & Verum (IR-A) & 20 & 66.5 & 12 & 8 & 10.3 & 185 & 17.9 \\
\hline & Placebo & 12 & 68.6 & 6 & 6 & 9.8 & 166 & 16.9 \\
\hline & No Irradiation & 24 & 66.0 & 12 & 12 & no & no & no \\
\hline \multirow[t]{4}{*}{ Low back pain } & Total group & 59 & 49.7 & 21 & 38 & & & \\
\hline & Verum (IR-A) & 32 & 48.3 & 11 & 21 & 9.3 & 149 & 16.0 \\
\hline & Placebo & 11 & 46.8 & 4 & 7 & 9.4 & 160 & 17.0 \\
\hline & No Irradiation & 16 & 54.5 & 6 & 10 & no & no & no \\
\hline Rheumatoid & Total group & 42 & 61.3 & 11 & 31 & & & \\
\hline Arthritis (non- & Verum (IR-A) & 17 & 56.1 & 5 & 12 & 10.1 & 158 & 15.7 \\
\hline inflammatory & Placebo & 8 & 63.8 & 2 & 6 & 10.2 & 162 & 15.9 \\
\hline period) & No Irradiation & 17 & 65.4 & 4 & 13 & no & no & no \\
\hline
\end{tabular}


Evaluation of pain degree and mobility measurements. Before the first treatment, after one week, and after two weeks of treatment the treating physiotherapists measured the mobility of joints or lumbar spinal cord which were treated and asked the patient to evaluate the pain degree according to a visual analog pain scale (VAS). The numbers 0 to 10 of the VAS correspond to: 0 "no pain", 10 "highest pain which is unbearable". Furthermore, therapeutic schedule, the use of analgetics and other drugs, and evaluation of the patient's subjective feeling were registered. The mobility of the joints was analyzed according to the neutral zero-method. The so-called normal physiological values of healthy persons were taken as hundred percent, e.g., for the hip $15 / 0 / 130-140^{\circ}$ for extension and flexion, 30-45/0/20$30^{\circ}$ for abduction and adduction, 30-50/0/30-50 for outer rotation and inner rotation (Wuelker, 2005), and for the knee 10-5/0/120-150 for extension and flexion (Wuelker, 2005).

The mobility of the lumbar vertebral column was analyzed according to the Schober sign, whose value is normal if it increases from $10 \mathrm{~cm}$ to $15 \mathrm{~cm}$ during flexion of the vertebral column. Therefore the difference of $5 \mathrm{~cm}$ corresponds to $100 \%$ (Wuelker, 2005). The physiological value of lateral flexion of the vertebral column (lumbar spinal cord) was set as $30 / 0 / 30^{\circ}$ being equal to $100 \%$.

MDA measurement. MDA was determined according to Wong et al. (1987) with modifications of Sommerburg et al. (1993) as the thiobarbituric acid (TBA) derivative. Phosphoric acid (440 mM), sample or MDA standard, and TBA solution (42 mM) were incubated at $100^{\circ} \mathrm{C}$ for $60 \mathrm{~min}$ and then the samples and standards were cooled on ice. To neutralize the phosphoric acid and to precipitate the proteins before the sample was injected into an HPLC system, the samples and standards were diluted 1:1 (v/v) with $\mathrm{NaOH}(0.1 \mathrm{M})$ in methanol. Afterwards, all samples were centrifuged at $10000 \times g$ for $2 \mathrm{~min}$. Aliquots of the derivatized samples were injected into a reversed phase HPLC system (HPLC from Shimadzu; C18 column from Supelco, $125 \mathrm{~mm} \times 4.6 \mathrm{~mm}$ ) and separated by isocratic elution with phosphate buffer (50 mM, pH 6.8) containing $40 \%$ (v/v) methanol. The TBA-MDA complex was detected by means of fluorescence using an excitation wavelength of $525 \mathrm{~nm}$ and emission of $550 \mathrm{~nm}$.

Statistics. After collection of data on joint or vertebral column mobility and pain intensity we asked, wheth-

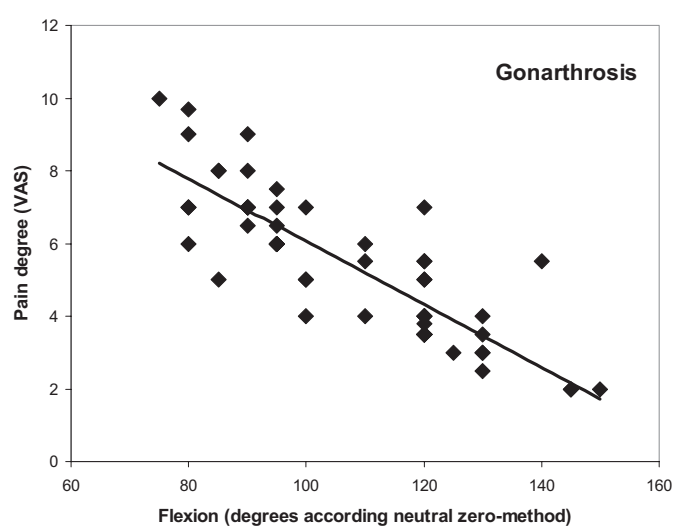

Figure 1. Linear correlation between initial (before first IR treatment) mobility reduction (flexion) and initial pain intensity in gonarthrosis.

Correlation coefficient $r$ was 0.8664 with error probability $P<0.0001$. er a statistically significant correlation exists between the mobility reduction and the pain intensity. Normal distribution of the data was examined by the KolmogorovSmirnov test. For statistical evaluation of time-dependent changes first the Friedman test was performed. The Wilcoxon rank test followed a positive outcome of the Friedman test. For examining significance of differences between the patient groups (Verum vs. Placebo, Verum vs. Non Irradiated, and Placebo versus Non Irradiated) the Kruskal-Wallis test for multiple independent samples was performed follwed by the Mann-Whitney U-test for independent samples. For normally distributed data (MDA levels), Student's double sided $t$-test for independent samples as well as ANOVA with Bonferroni post-hoc testing for significant differences was performed. In addition, correlation analysis based on Spearmans's rho was also performed. Changes and correlations were evaluated to be statistically significant if the probability of error $P$ was less than 0.05 .

\section{RESULTS}

\section{Correlation between mobility reduction and pain intensity}

Correlation analysis showed a significant negative correlation (Spearman's rho) of initial mobility reduction with initial pain intensity in gonarthrosis $(-0.855$; $P<0.001)$, coxarthrosis $(-0.634 ; P<0.001)$, and RA patients $(-0.540 ; P<0.005)$, and borderline significant one in patients suffering from low back pain $(-0.261$; $P=0.061$ ). The correlation among initial (before first IR treatment) mobility reduction and the initial pain intensity follows a linear regression model, however, the correlation coefficients $(r)$ and the corresponding error probability $(P)$ differ for different diagnosis groups. Figure 1 shows this correlation for patients suffering from gonarthrosis with $r=0.8664$ and $P<0.0001$. The $r$ and $P$ for other groups were $r=0.5800$ and $P<0.0001$ for coxarthrosis, $r=0.3476$ and $P=0.018$ for low back pain syndrome, and $r=0.5920$ and $P=0.0016$ for RA. Furthermore, it was found that there were no significant differences between the initial pain intensities in the different patient groups investigated. Apparently, suffering from degenerative joint or lumbar spinal cord diseases produces the same subjective "degree of suffering" as does RA.

\section{Degenerative osteoarthritis: coxarthrosis and gonarthrosis}

In the Verum group of patients suffering from coxarthrosis there was a significant increase (always with $P<0.01)$ of hip mobility after the first and after the second week of IR-A treatment, which is shown for hip flexion in Fig. 2 (left-hand scale and continuous lines). The hip mobility values were significantly different between time zero and one week, time zero and two weeks, and between one week and two weeks $(P<0.05)$. Furthermore, there was a significant pain reduction in the Verum group according to the Wilcoxon rank test following a positive Friedman test, which is also shown in Fig. 2 (right-hand scale and dashed lines) with significant differences between all three time points. Neither for the mobility nor for the pain were the score changes in the Placebo and No Irradiation groups significant. 


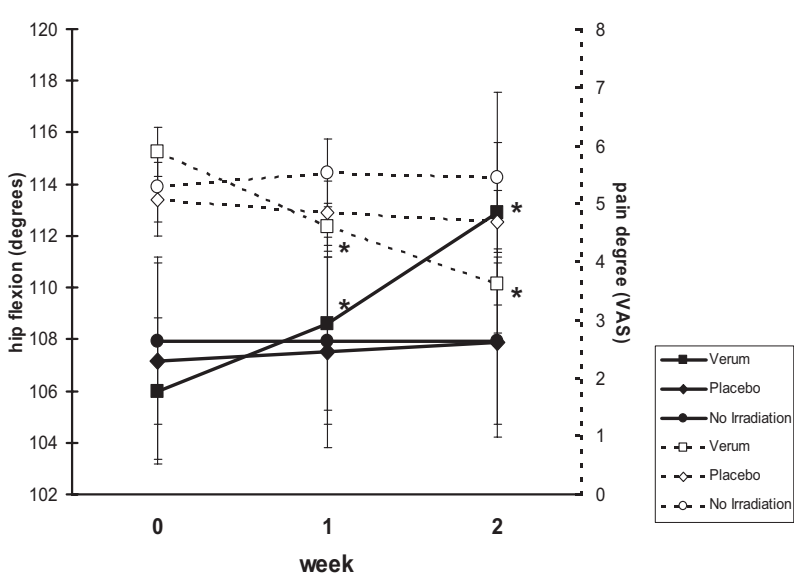

Figure 2. Hip flexion and hip pain in coxarthrosis patients Flexion as most important mobility parameter is shown by continuous lines. Pain degree as measured with VAS is shown by dashed lines. Number of patients was 19 in Verum, 14 in Placebo, and 17 in No Irradiation group. Significances for changes in both mobility/flexion and pain degree: ${ }^{*} P<0.05$ and ${ }^{* *} P<0.005$ compared with corresponding pre-treatment (0 week) (Friedmann statistics); in Placebo group and No Irradiation groups no significant differences.

Similar results were obtained for gonarthrosis. Fig. 3 shows the changes in knee mobility/flexion (left-hand scale and continuous lines) and knee pain degree (righthand scale and dashed lines). Also here in the Verum group of patients the mobility and pain improved already after one week of therapy (all improvements were statistically significant with $P<0.01)$, the differences to the Placebo and No Irradition group being significant $(P<0.05 ; P<0.005)$. Taking hip and knee patients with degenerative osteoarthritis together (in total one-hundred and six patients), the average pain score after two weeks of InfraCare treatment was reduced by $1.4 / 10$ after one week, and by 2.2/10 after two weeks (Fig. 4). In addition, significant $(P<0.005)$ differences, albeit minor, were also seen in the Placebo group compared

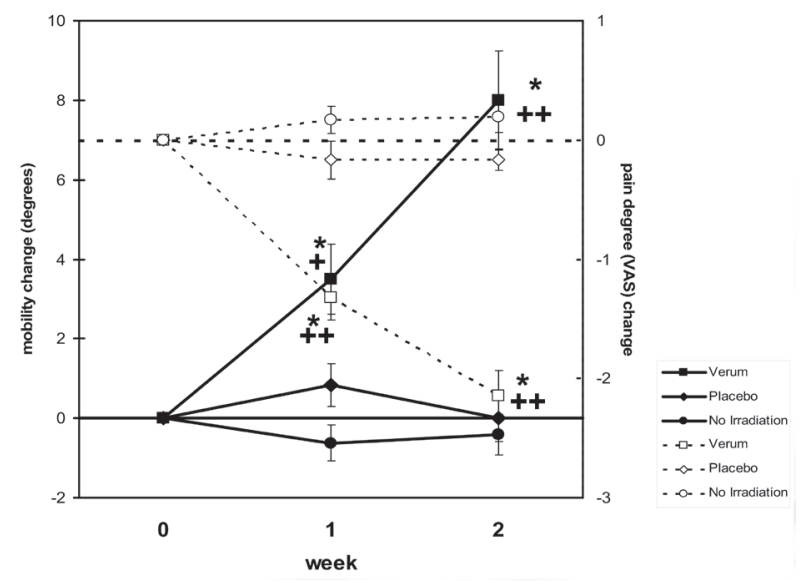

Figure 3. Changes of knee flexion and knee pain in gonarthrosis patients

Changes of knee flexion are given by continuous lines. Changes of pain degree as measured with VAS are shown by dashed lines. Number of patients was 20 in Verum, 12 in Placebo, and 24 in No Irradiation group. ${ }^{*} P<0.05$ and ${ }^{*} P<0.005$ compared with 0 weeks (Friedmann statistics); in Placebo and No Irradiation no significant differences. $+P<0.05$ and $++P<0.005$ compared with Placebo and No Irradiation group at the corresponding timepoints (Mann-Whitney-U statistics).

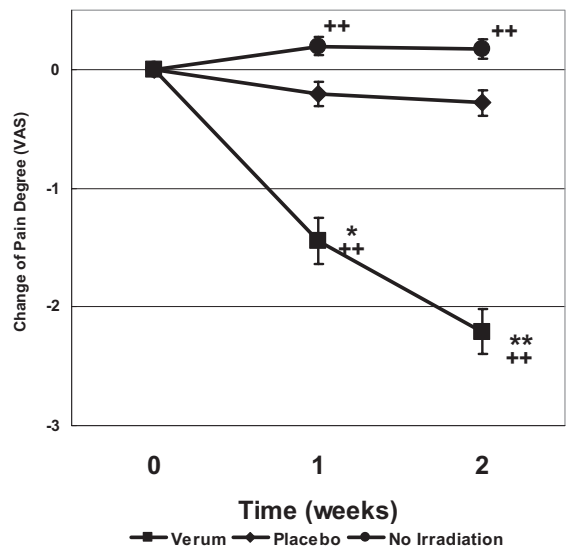

Figure 4. Pain reduction in patients suffering from degenerative osteoarthritis of hip or knee

Coxarthrosis and gonarthrosis patients are taken together (in total 106 patients). Verum group $(n=39)$, Placebo group $(n=26)$, and No Irradiation group $(n=41)$ as for Figs. 2 and 3. Significant changes: ${ }^{*} P<0.05$ and ${ }^{* *} P<0.005$ compared with 0 weeks (Friedmann statistics); $++P<0.005$ compared with Placebo group at the corresponding timepoints (Mann-Whitney-U statistics); Note: $P<0.005$ holds also true for Verum versus No Irradition at both timepoints.

to the No Irrradiation group. Furthermore, significant $(P<0.05)$ functional improvement was measured after two weeks of treatment in the Verum group in comparison with the Placebo and No Irradiation groups, too, but it was minor. However, the major IR-A effect after already one and - to a greater extent — after two weeks of treatment was the pain relief, which is demonstrated in Fig. 4.

\section{Low back pain}

In patients with low back pain the treatment led to a significant increase of mobility (Fig. 5; left-hand scale, thick lines). The significance was checked using the Wilcoxon rank test following a positive Fried-

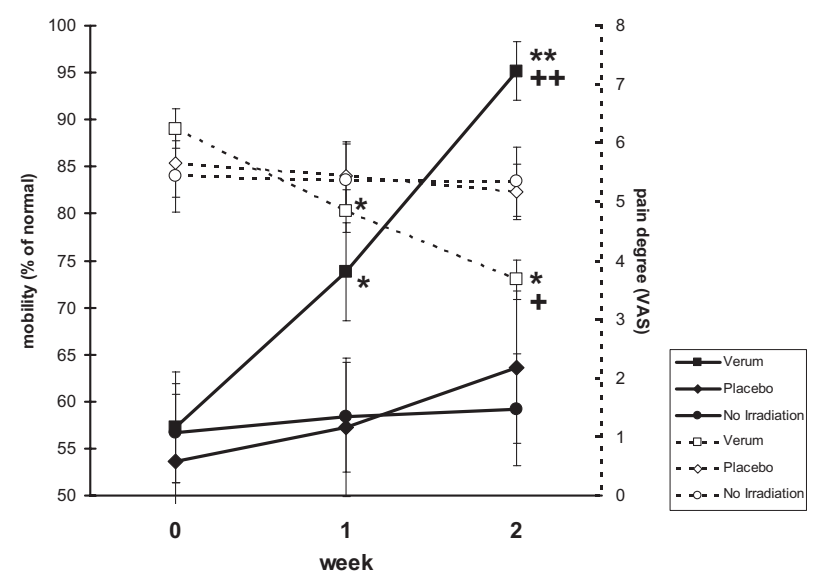

Figure 5. Mobility and pain improvement in patients with low back pain

Vertebral column mobility (\% of normal mobility measured according to Schober sign) is given by continuous lines. Pain degree (values according to Visual Analogue Scale) is given by dashed lines. Number of patients was 32 in Verum, 11 in Placebo, and 16 in No Irradiation group. ${ }^{*} P<0.05$ and ${ }^{*} * P<0.005$ compared with 0 weeks (Friedmann statistics); in Placebo and No Irradiation no significant differences. $+P<0.05$ and $++P<0.005$ compared with Placebo and No Irradiation group at the corresponding timepoints (Mann-Whitney-U statistics) 


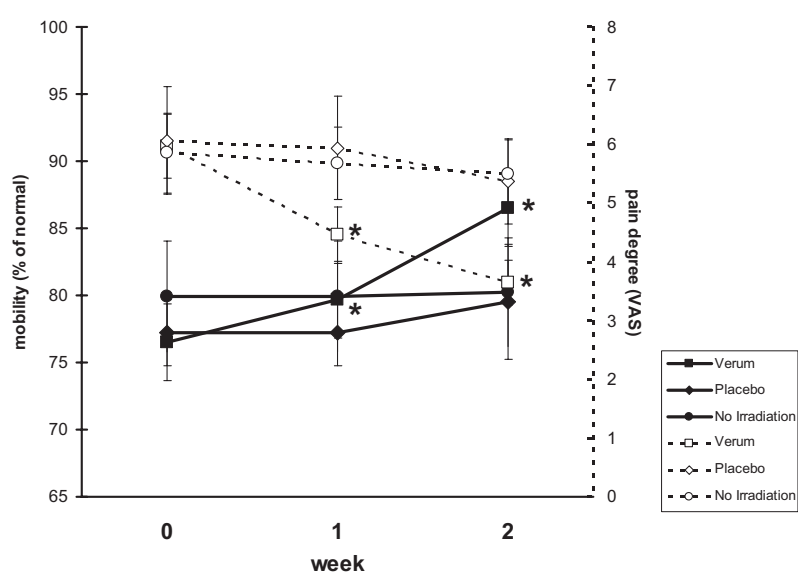

Figure 6. Mobility and pain improvement of disease leading joint of patients suffering from rheumatoid arthritis (RA) Mobility is shown by continuous lines, pain by dashed lines. Number of patients was 17 in Verum, 8 in Placebo, and 17 in No Irradiation group. ${ }^{*} P<0.05$ compared with 0 weeks (Friedmann statistics); in Placebo and No Irradiation no significant differences.

man test in the Verum group between all time points. There were no significant changes in the Placebo or No Irradiation groups. Also in this disease a significant pain reduction was found in the Verum group, whereas there were no significant pain improvements in the other groups (Fig. 5; right-hand scale, dashed lines), the differences between Verum and Placebo as well as No Irradiation group being significant $(P<0.005)$ after two weeks. Taking the data of the Verum group together, both the improvement of mobility and pain reduction were by about 35-40\%.

\section{Rheumatoid arthritis (RA)}

Clinical improvements in 42 patients suffering from RA were investigated after two weeks of treatment. There was a significant increase of mobility of the disease leading joint in the Verum group, with a smaller increase within the first week $(P<0.05)$ and a stronger one during the second week $(P<0.01$ for Verum zero vs. Verum 2 weeks) (Fig. 6). There were no significant changes in the Placebo or No Irradiation groups. The Verum group, furthermore, experienced a significant pain reduction (Fig. 6), also without significant changes in the other groups. The pain reduction was the major clinical effect, whereas the improvement of joint mobility was a minor, but also significant, one.

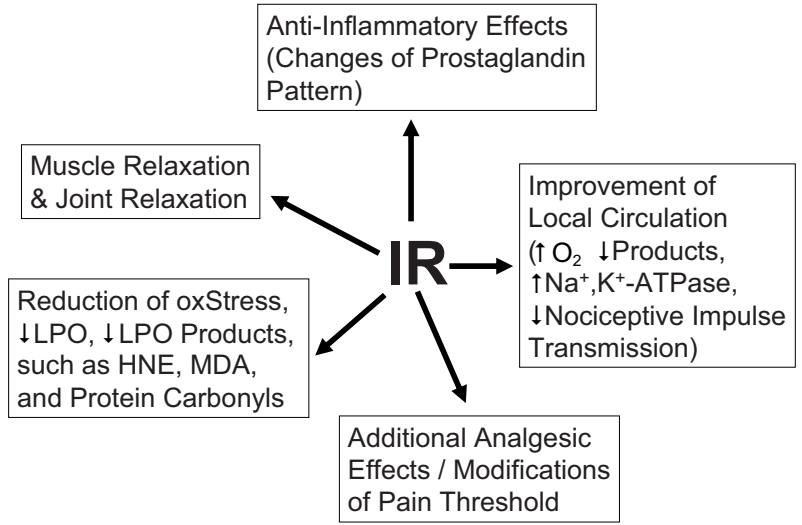

Figure 7. Schematic representation of biological effects of warmth and in particular IR-A irradiation contributing to pain relief (see text)

\section{Malondialdehyde (MDA) serum concentration}

Table 2, shows that the mean values of serum MDA are in the range between $0.5 \mu \mathrm{M}$ and $0.85 \mu \mathrm{M}$. Interestingly, the mean values were significantly $(P<0.05$, $P<0.005)$ lower in the group of patients suffering from low back pain before and after IR-A treatment compared with the other disease states. Upon IR-A irradiation, the MDA levels significantly $(P<0.05)$ decreased in low back pain and rheumatoid arthritis patients, but remained unchanged in the osteoarthrosis group.

\section{DISCUSSION}

\section{Indications for IR-A therapy}

A multitude of conditions have been suggested as indications for IR therapy (Hammond, 2004; Baronzio et al., 2006; Kukkonen-Harjula \& Kauppinen, 2006; Perret et al., 2006), such as a) non-inflammatory pain in degenerative diseases of the vertebral column, low back pain, fibromyalgia, degenerative joint diseases, such as gonarthrosis, coxarthrosis, omarthrosis, traumatic fibrosis, Xray-induced fibrosis, scar treatment, sprains, bruises, b) chronic inflammatory diseases of the skin, mucosal tissues, bones, joints, chronic bronchitis, sinusitis, prostatitis, gynecological diseases (not during acute inflammatory periods), c) hypertonus, and d) allergic diseases such as urticaria, pollinosis, and asthma bronchiale. It is needless

Table 2. MDA serum levels before and after two weeks of infrared radiation treatment

\begin{tabular}{llrrr}
\hline Diagnosis group & Subgroup & MDA pre $[\mu \mathrm{M}]$ & MDA post $[\mu \mathrm{M}]$ & Diff. $[\mu \mathrm{M}]$ \\
\hline Gonarthrosis & Verum ${ }^{2}$ & $0.76 \pm 0.14(20)$ & $0.71 \pm 0.18(20)$ & $-0.05 \pm 0.12(20)$ \\
& Placebo & $0.73 \pm 0.12(12)$ & $0.74 \pm 0.11(12)$ & $0.01 \pm 0.12(12)$ \\
Coxarthrosis & Verum & $0.67 \pm 0.14(19)$ & $0.63 \pm 0.12(19)$ & $-0.04 \pm 0.08(19)$ \\
& Placebo & $0.69 \pm 0.12(14)$ & $0.70 \pm 0.12(14)$ & $0.01 \pm 0.12(14)$ \\
Low Back & Verum & $0.58 \pm 0.12(32)^{+}$ & $0.53 \pm 0.09(32)^{++}$ & $-0.05 \pm 0.07(32)^{*}$ \\
Pain & Placebo & $0.53 \pm 0.06(11)^{++}$ & $0.55 \pm 0.08(11)^{++}$ & $0.02 \pm 0.07(11)$ \\
RA $^{3}$ & Verum & $0.85 \pm 0.15(17)$ & $0.75 \pm 0.11(17)$ & $-0.10 \pm 0.09(17)^{*}$ \\
& Placebo & $0.80 \pm 0.07(8)$ & $0.78 \pm 0.06(8)$ & $-0.02 \pm 0.09(8)$ \\
\hline
\end{tabular}

${ }^{1}$ Diff $=$ MDA post - MDA pre (negative values indicate MDA reduction). Values represent the mean \pm S.D. Sample size is given in parentheses. ${ }^{2}$ Verum $=\mathrm{IR}$ treated. ${ }^{3}$ Rheumatoid arthritis - non-inflammatory period. ${ }^{*} P \leq 0.05$ compared with the corresponding Placebo group; Student's double sided $t$-test for independent samples.; $+P \leq 0.05,++P \leq 0.005$ compared with the corresponding MDA levels found in Gon-/Coxarthrosis and RA; ANOVA (Bonferroni post-hoc testing). 
to mention that effective prevention, treatment and rehabilitation of musculoskeletal pain presents a major economic factor in all countries.

\section{Effects of warmth therapy and in particular IR-A irradiation}

In Fig. 7 further factors contributing to pain relief are summarized. In general, warmth therapy works via direct relaxation of the muscles and via thermoregulation. The thermo-regulatory effects include the body reacting by increasing the blood circulation to cool down when the temperature gets above the body core temperature $\left(37^{\circ} \mathrm{C}\right)$. The improved blood circulation leads to increased oxygen and nutrient supply. Furthermore, it also accelerates the removal of metabolic end-products and carbon dioxide from muscle tissue. Anti-inflammatory effects due to changes of prostaglandin pattern of irradiated tissue have also been described (Hardy et al., 1951; Moriyama et al., 2005; Pulichino et al., 2006; Rosseland et al., 2007). It is known that under conditions of increased or normalized tissue $\mathrm{pO}_{2}$ and increased substrate supply the activity of $\mathrm{Na}^{+}-\mathrm{K}^{+}$-ATPase increases or, at least, does not decrease. A high $\mathrm{Na}^{+}-\mathrm{K}^{+}$-ATPase activity correlates with decreased nociceptive impulse transmission (Moriyama et al., 2005). Classic reports exist on modification of pain threshold by warmth and IR irradiation (Khanapure et al., 2007). Additionally, data of our research group indicate a reduction of oxidative stress during warmth therapy. In sauna therapy a reduced lipid peroxidation (LPO) rate and reduced plasma concentrations of toxic aldehydic LPO products such as malondialdehyde (MDA), 4-hydroxy-2,3-trans-nonenal (HNE) and protein carbonyls were measured (Gruenberger, 2007). It is known that MDA and HNE can modify proteins and nucleic acids. The reduction of $\mathrm{Na}^{+}-\mathrm{K}^{+}-\mathrm{A}$ TPase activity, an enzyme which obviously relates to nociception, by aldehydic LPO products was described in detail (Siems et al., 1996; 2003).

\section{Equipment with high content of IR-A irradiation}

The devices of the InfraCare series produce IR with $45-50 \%$ of IR-A. The naturally low IR-A absorption at the skin surface (IR-A penetrates the skin up to a depth of $5 \mathrm{~mm}$ to $6 \mathrm{~mm}$, whereas IR-B and IR-C have maximal penetration depths of $2.5 \mathrm{~mm}$ or even $0.2 \mathrm{~mm}$ to $0.3 \mathrm{~mm}$, respectively (Piazena, 2002)) and the low contents of IR-B and IR-C which are absorbed near the surface guarantee a warmth-induced wellbeeing far from the heat pain threshold. This allows longer periods of irradiation and a high intensity of irradiation with the aim of accelerated circulation, which is only possible by IR-A. The devices are safe and easy to handle. Another advantage is the homogeneity of irradiation without hot spots. The optimal application time is 15 min. Warnings, recommendations of the producer, and proposed contraindications were described under Material and Methods. In this study no single case of side effects occurred.

\section{Clinical use of IR-A irradiation}

The investigations showed clinical improvements in four diseases: degenerative osteoarthritis of the hip and of the knee, i.e. in coxarthrosis and gonarthrosis, low back pain, and rheumatoid arthritis. The clinical improvements occur already after short periods of application, i.e. after one and two weeks. Long-term effects were observed only in selected patients, but should be investigated in a further study.

In patients suffering from gonarthrosis or coxarthrosis pain reduction according to the Visual Analogue Scale evaluation (by about 2 points) and functional improvement were measured after two weeks of treatment in the Verum group in comparison with the Placebo and No Irradiation groups. Data from a randomized double-blind study were previously available only for gonarthrosis patients. In that study by Stelian et al. (1992) significant pain reduction and mobility improvement was found in the IR, but not in the Placebo group after 20 treatments within ten days (Stelian et al., 1992). In our study in gonarthrosis patients similar effects were seen with the modern devices already with ten applications (see Table 1).

In low back pain both the improvement of mobility and pain reduction were by about $35-40 \%$ in the Verum (IR-A) group.

Clinical improvements were also seen in patients suffering from rheumatoid arthritis.

In general, pain reduction was the major clinical effect, whereas the improvement of joint or vertebral column mobility was minor, but also significant. The data on pain reduction were indeed striking, with an improvement of pain degree by 2 points or more reported by $75 \%$ of gonarthrosis, $63 \%$ of coxarthrosis, $69 \%$ of degenerative osteoarthritis patients together, $72 \%$ of low back pain, and $71 \%$ of rheumatoid arthritis patients. For a pain relief by at least 1.5 points after IR-A treatment, the corresponding proportions of patients were as high as $90 \%$ (gonarthrosis), $84 \%$ (coxarthrosis), $87 \%$ (gonarthrosis and coxarthrosis together), $81 \%$ (low back pain), and $82 \%$ (RA).

\section{Measurement of lipid peroxidation by means of MDA}

The serum MDA concentrations in patients of the three diagnosis groups were in the range of physiological values of age-matched healthy population (Gil et al., 2006). Interestingly, the mean values were lower in the group of patients suffering from low back pain before and after IR-A treatment compared with the other disease states. The MDA values in osteoarthrosis and rheumatoid arthritis, which are in the upper range of normal values, demonstrate that during non-inflammatory periods of these diseases little lipid peroxidation occurs. However, during inflammatory periods high generation of reactive oxygen species and lipid peroxidation products takes place. There were small but significant decreases of the MDA levels upon IR-A irradiation in low back and rheumatoid arthritis patients. MDA remained unchanged upon IR-A irradiation in the osteoarthrosis group. However, in all diagnosis groups the pain intensity decreased substantially upon irradiation. Obviously MDA is not a sensitive biomarker for the reflection of rapid pain reduction, even though a reduction of lipid peroxidation could be expected. In addition, the data show that there is no increase of in vivo lipid peroxidation by intensive IR-A-treatment. That confirms the classical opinion that IR radiation is harmless, but argues against previous reports on significant dangers by IR radiation. Previously it was reported that IR-A induces expression of matrix metalloproteinase-1 (MMP1 ), which was found to be mediated by the formation of intracellular reactive oxygen species (Schroeder et al., 2007; 2008). The data shown here at least argue against a strong impact of this subcellular phenomenon in the cir- 
culating blood or in the whole organism. The unchanged or even decreased MDA serum levels show that intensive IR-A irradiation, as carried out here, does not lead to increased oxidative stress.

Taken together, the data strongly suggest IR-A irradiation as an effective and harmless method in prevention, therapy and rehabilitation of degenerative osteoarthritis, low back pain, and during non-inflammatory periods of RA.

\section{REFERENCES}

Bagis S, Tamer L, Sahin G, Bilgin R, Guler H, Ercan B, Erdogan C (2005) Free radicals and antioxidants in primary fibromyalgia: an oxidative stress disorder? Rheumatol Int 25: 188-190.

Baronzio G, Gramaglia A, Fiorentini G (2006) Hyperthermia and immunity. In Vivo 20: 689-695.

Gil L, Siems W, Mazurek B, Gross J, Schroeder P, Voss P, Grune T (2006) Age-associated analysis of oxidative stress parameters in human plasma and erythrocytes. Free Radic Res 40: 494-505.

Gruenberger P (2007) Oxidativer Stress bei thermischen Ganzkörperreizen - Sauna im Vergleich zum Winterschwimmen M.D. Dissertation, Humboldt UniversityPress, Berlin.

Hammond A (2004) Rehabilitation in rheumatoid arthritis: a critical review. Musculoskeletal Care 2: 135-151.

Hardy JD, Goodell H, Wolff HG (1951) The influence of skin temperature upon the pain threshold as evoked by thermal radiation. Science 114: 149-150.

Khanapure SP, Garvey DS, Janero DR, Letts LG (2007) Eicosanoids in inflammation: biosynthesis, pharmacology, and therapeutic frontiers. Curr Top Med Chem 7: 311-340.

Kukkonen-Harjula K, Kauppinen K (2006) Health effects and risks of sauna bathing. Int J Circumpolar Health 65: 195-205.

Masuda A, Koga Y, Hattanmaru M, Minagoe S, Tei C (2005) The effects of repeated thermal therapy for patients with chronic pain. Psychother Psychosom 74: 288-294.

Merskey H, Watson GD (1979) The lateralisation of pain. Pain 7: 271280.

Merskey H (1989) Psychiatry and chronic pain. Can J Psychiatry 34: 329-336.

Moriyama T, Higashi T, Togashi K, Iida T, Segi E, Sugimoto Y, Tominaga T, Narumiya S, Tominaga M (2005) Sensitization of TRPV1 by EP1 and IP reveals peripheral nociceptive mechanism of prostaglandins. Mol Pain 17: 3.

Ozgocmen S, Ozyurt H, Sogut S, Akyol O, Ardicoglu O, Yildizhan H (2006) Antioxidant status, lipid peroxidation and nitric oxide in fibromyalgia: etiologic and therapeutic concerns. Rheumatol Int 26: 598-603.
Perret DM, Rim J, Cristian A (2006) A geriatrician's guide to the use of the physical modalities in the treatment of pain and dysfunction. Clin Geriatr Med 22: 331-354.

Piazena H (2002) Nutzung künstlich erzeugter Infrarot-Strahlung für Prophylaxe und Wellness - Grundlagen, Grenzwerte und Qualitätskontrolle. Saunabetr Baederpraxis 1: 13-17.

Pulichino AM, Rowland S, Wu T, Clark P, Xu D, Mathieu MD, Riendeau D, Audoly LP (2006) Prostacyclin antagonism reduces pain and inflammation in rodent models of hyperalgesia and chronic arthritis. J Pharmacol Exp Ther 319: 1043-1050.

Rokyta R, Holecek V, Pekarkova I, Krejcova J, Racek J, Trefil L, Yamamotova A (2003) Free radicals after painful stimulation are influenced by antioxidants and analgesics. Neuro Endocrinol Lett 24: 304-309.

Rosseland LA, Krohn CD, Stubhaug A (2007) Synovial fluid concentration of prostaglandin E2 correlates with pain intensity after knee arthroscopic procedures. Anesth Analg 104: 460-461.

Schroeder P, Lademann J, Darvin ME, Stege H, Marks C, Bruhnke S, Krutmann J (2008) Infrared radiation-induced matrix metalloproteinase in human skin: implications for protection. I Invest Dermatol 128: 2491-2497.

Schroeder P, Pohl C, Calles C, Marks C, Wild S, Krutmann J (2007) Cellular response to infrared radiation involves retrograde mitochondrial signalling. Free Radic Biol Med 43: 128-135.

Siems WG, Hapner SH, van Kuijk F (1996) 4-Hydroxynonenal inhibits $\mathrm{Na}^{+}-\mathrm{K}^{+}$-ATPase. Free Radical Biol Med 20: 215-223.

Siems W, Capuozzo E, Lucano A, Salerno C, Crifò C (2003) High sensitivity of plasma membrane ion transport ATPases from human neutrophils towards 4-hydroxy-2,3-trans-nonenal. Life Sciences 73: 2583-2590.

Smith HS (2006) Arachidonic acid pathways in nociception. I Support Oncol 4: 277-287.

Sommerburg O, Grune T, Klee S, Ungemach FR, Siems WG (1993) Formation of 4-hydroxynonenal and further aldehydic mediators of inflammation during bromo-trichloromethane treatment of rat liver cells. Mediat Inflamm 2: 27-31.

Sondik EJ, Madans JH, Sadagursky MH (2005) Vital and Health Statistics Series 10, no. 225, Summary Health Statistics for U.S. Adults: National Health Interview Survey 2003, National Center for Health Statistics, Hyatsville, Maryland, DHHS Publication No. (PHS) 2005 1553: 1-151.

Stelian J, Gil I, Habot B, Rosenthal M, Abramovici I, Kutok N, Khahil A (1992) Improvement of pain and disability in elderly patients with degenerative osteoarthritis of the knee treated with narrow-band light therapy. J Amer Geriatrics Soc 40: 23-26.

Stewart WG, Ricci JA, Chee W, Morganstein D, Lipton R (2003) Lost productive time and cost due to common pain conditions in the US workforce. JAMA 290: 2443-2454.

Wong SHJ, Knight JA, Hopfer SM, Zaharia O, Leach CN, Sunderman FW (1987) Lipoperoxides in plasma as measured by liquid-chromatographic separation of malondialdehyde-thiobarbituric acid adduct. Clin Chem 33: 214-220.

Wuelker N (2005) Orthopaedics and traumatological surgery. Georg Thieme Verlag, Stuttgart, New York. 